\title{
Nail Changes Due To Docetaxel Therapy: Case Report
}

\author{
Amorim GM ${ }^{1,2 *}$, Amorim DLV ${ }^{2}$, Amorim $G^{3}$ and Amorim-filho RM $\mathbf{M}^{3,4}$ \\ ${ }^{1}$ Clementino Fraga Filho University Hospital, Federal University of Rio de Janeiro \\ ${ }^{2}$ Institute of Dermatology Professor Rubem David Azulay, Santa Casa de Misericórdia of Rio de Janeiro
}

${ }^{3}$ University of Southern Santa Catarina

${ }^{4}$ Private clinic

Received: October 18, 2017; Accepted: November 03, 2017 ; Published: November 10, 2017

*Corresponding author: Amorim GM, Clementino Fraga Filho University Hospital, Federal University of Rio de Janeiro, Brazil, E-mail: gustavomoreiraamorim@hotmail.com

\begin{abstract}
We report a case of 72 years old man, with prostate cancer, under chemotherapy treatment with docetaxel, referred to the dermatologist by the assistant oncology due to painful ungual lesions. The dermatological exam showed onycholysis, subungual hemorrhages and subungual abscess, affecting most of his fingernails. Since the ungual changes were secondary to docetaxel, the relevant literature was reviewed and it is presented in this case report.
\end{abstract}

\section{Introduction}

Chemotherapy is defined as the method that uses chemicals in the treatment of diseases caused by biological agents. When applied in order to treat malignant neoplastic diseases, it is called antineoplastic chemotherapy. The antineoplastic agents included in this modality of treatment, in general acts inhibiting or preventing the proliferation of neoplastic cells $[1,2]$.

Drugs used in antineoplastic chemotherapy interfere with the proliferation function of both normal and neoplastic cells, where differences in replication rates and cell biochemistry itself drive a greater performance from the antineoplastic agent on neoplastic cell clones. However, as mentioned, normal cells also suffer some type of alteration due to the action of the chemotherapeutic drug $[3,4]$.

The skin, mucosal membranes and cutaneous appendages, due to their high metabolic rates, often suffer some effect directly related to the toxicity of an antineoplastic chemotherapeutic agent $[1,2,4]$.

The correct interpretation of a cutaneous adverse reaction to a given chemotherapeutic agent or even a chemotherapeutic group of agents is very important, and the dermatologist should be careful in the evaluation of patients under treatment. The dermatologist should always take into account the differential diagnosis of infectious diseases, given the immunosuppression of this group of patients, or even the cutaneous manifestations of the underlying neoplastic disease itself [1-4].
We present a case of a male patient with prostatic adenocarcinoma undergoing chemotherapy, presenting recently with nail complaints. The objective of this report is to draw attention to the correlation between the antineoplastic agent and the nail findings presented, given the increasing number of patients diagnosed with cancer, being treated with chemotherapy.

\section{Case Report}

A 72-year-old male patient, retired, with a prostatic adenocarcinoma, being treated regularly with docetaxel over the last 4 months, presented for a dermatological consultation complaining of nail alterations. Three weeks ago he started with painful lesions on his fingernails, bilaterally. He denied any history of local trauma.

Dermatological examination showed: irregular distal onycholysis on the 2nd, 3rd, 4th and 5th fingernail of the right hand and $2 \mathrm{nd}$, 3rd and 4 th on the left hand, associated with yellowish-brown chromonychia; Subungual hemorrhage on the 3rd right hand fingernail and on the 3rd of left, as well as Subungual abscess on the 4th right hand fingernail (Figures 1, 2 and 3). Significant pain was reported during the exam.

Since our diagnostic hypothesis was a side effect of the chemotherapy treatment, the patient was treated with drainage of the hematomas and abscess, associated with warm compresses and oral analgesics, for symptomatic relief. After discussion with the assistant oncologist and in agreement with the patient desire, it was decided to maintain the treatment. Patient is still being followed.

\section{Discussion}

Docetaxel, along with paclitaxel, is cytotoxic chemotherapeutic agents that comprise the class of taxanes, which are among the most commonly prescribed chemotherapy drugs in oncology. Paclitaxel is a natural compound extracted from the bark of a Pacific tree whose species is called Taxus brevifolia. Docetaxel is a semi-synthetic analog of paclitaxel. Both drugs 


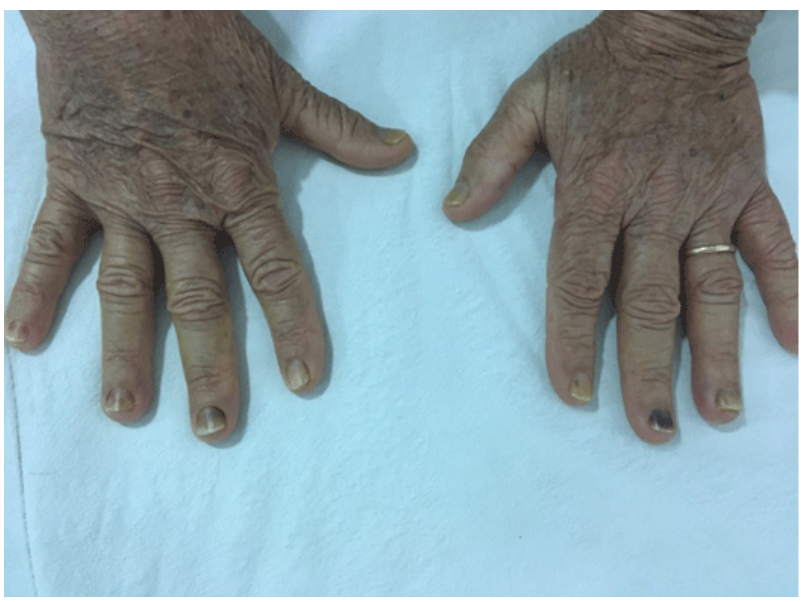

Figure 1: Irregular distal onycholysis on the $2 \mathrm{nd}, 3 \mathrm{rd}$, 4 th and 5 th fingernail of the right hand and 2nd, 3rd and 4th on the left hand, associated with yellowish-brown chromonychia.

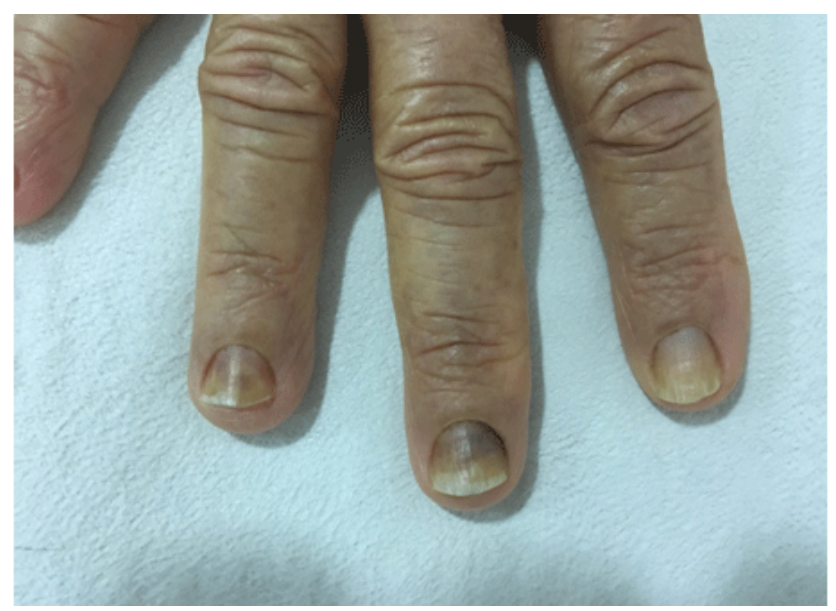

Figure 2: Subungual hemorrhage on the 3rd and subungual abscess on the 4 th right hand fingernail.

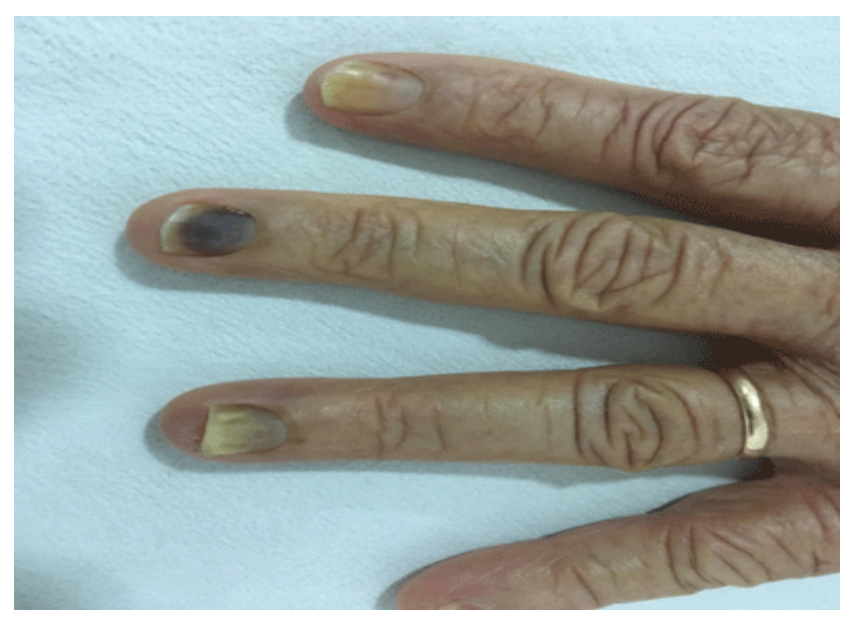

Figure 3: Subungual hemorrhage on the 3rd left hand fingernail. act as antimicrotubule agents, promoting the polymerization of intracellular microtubules, interrupting the process of mitosis and leading to cell death [3].

According to the Food and Drugs Administration (FDA), taxanes (paclitaxel and docetaxel) are indicated for the following cancers: breast (as adjuvant in metastatic disease), non-small cells lung cancer, ovary, prostate (with androgen independent metastases), stomach, Kaposi sarcoma and some types of head and neck cancer [5].

The cutaneous adverse effects of treatment with the taxane class of chemotherapeutic are common, affecting, to some degree, the majority of patients under treatment; even though real incidence is unknown. Docetaxel appears to be more associated than paclitaxel to cutaneous adverse effects. The table 1 , adapted from a recent review article on the dermatological side effects associated with taxanes, shows the main manifestations and their estimated occurrence [3].

Table 1: Ungual toxicities induced by paclitaxel and docetaxel.

\begin{tabular}{|l|c|}
\hline Appendages Changes & Global Incidence* \\
\hline Ungual changes: & $++/ 3$ \\
\hline -Onycholysis $* *$ & $+/ 3$ \\
\hline $\begin{array}{l}\text {-Other ungual alterations(Onychomadesis, Beau } \\
\text { lines, Melanonychia, Leukonychia, Paronychia, } \\
\text { Onychorrehxis) }\end{array}$ & + \\
\hline
\end{tabular}

*Expressed visually, with a crosses system gradation. Minimum: $+/ 3$ and maximum: $+++/ 3$.

**More associated with docetaxel. Adapted: Sibaud V, et al. Eur J Dermatol. 2016;26(5):427-443.

Nail changes are common during treatment with taxanes. A systematic review and meta-analysis investigated the risk of nail abnormalities associated with taxanes chemotherapy, published in 2015, found a global incidence of nail abnormalities in $43.7 \%$ in patients using paclitaxel and $34.9 \%$ with docetaxel. The relative risk of developing nail abnormalities during treatment with this class of drugs was 77.4 ( $p<0.001$ and 95\% CI 41.88 - 144.32) [6].

It is important to emphasize that paclitaxel and docetaxel are the chemotherapeutic drugs that most induce nail changes, but the mechanism of occurrence is still not well established. A direct cytotoxic effect on matrix cells or even bed epithelial cells and anti-angiogenic effect of taxanes are the current hypothesis [7].

Clinical manifestations can be originated on the nail matrix (melanonychia, true leuconychia, Beau lines, onychomadesis, onychorrhexis, pachyonychia and koilonychia), on the nail bed (onycholysis, apparent leukonychia) and on the nail fold (paronychia). These become generally apparent after a few weeks of treatment, given the slow rate of growth of the nail plate $[3,8]$.

Onycholysis is the most characteristic change due to docetaxel. The fingernails are more often affected in relation to the toenails. The onycholysis can be diffuse affecting several nails and is usually painful, being the discomfort directly related to the degree of detachment and the number of affected nails. It therefore determines a significant impairment of the quality of 
life $[8,9]$.

Subungual hematoma, hemorrhage and Subungual abscess are not uncommon, and can be debilitating $[8,10]$.

Management of nail changes secondary to taxane treatment should be individualized, taking into account the type and extent of the involvement, the degree of impairment on daily activities and, of course, the effectiveness of the treatment to the neoplastic disease. Thus, the definition of conduct takes into account the opinion of the dermatologist and the oncologist, always taking into consideration the patient and his desires.

\section{Conclusions}

With this illustrative case report we seek to draw the attention of the dermatologists to the increasingly frequent cutaneous adverse reactions to chemotherapy, due to the progressive aging of the population and the increase of cancer diagnoses, consequently, a greater number of patients undergoing chemotherapy. The possibility of occurrence of not only skin involvement, but also of its appendages is highlighted. It is crucial, therefore, that we always be vigilant when watching this particular population.

\section{References}

1. Sanches JA Jr, Brandt HRC, Moure EMD, Pereira GLS, Criado PR. Reações tegumentares adversas relacionadas aos agentes antineoplásicos Parte I. An Bras Dermatol. 2010;85(4):425-437.

2. Criado PR, Brandt HRC, Moure ERD, Pereira GLS, Sanches JA Jr. Reações tegumentares adversas relacionadas aos agentes antineoplásicos Parte II. An Bras Dermatol. 2010;85(5):591-608.
3. Sibaud V, Lebœuf NR, Roche H, Belum VR, Gladieff L, Deslandres M, et al. Dermatological adverse events with taxane chemotherapy. Eur J Dermatol. 2016;26(5):427-443.

4. Shi VJ, Levy LL, Choi JN. Cutaneous manifestations of nontargeted and targeted chemotherapies. Semin Oncol. 2016;43(3):419-425. doi: 10.1053/j.seminoncol.2016.02.018

5. Nacional Cancer Institute in the National Institute of Health (NIH). About cancer: treatment - A to Z list of Cancer Drugs. Available in: https://www.cancer.gov/about-cancer/treatment/drugs/fdadocetaxel and https://www.cancer.gov/about-cancer/treatment/ drugs/paclitaxel

6. Capriotti K, Capriotti JA, Lessin S, Wu S, Goldfarb S, Belum VR, et al. The risk of nail changes with taxane chemotherapy: a systematic review of the literature and meta-analysis. Br J Dermatol. 2015;173(3):842-855. doi: 10.1111/bjd.13743

7. Minisini AM, Tosti A, Sobrero AF, Mansutti M, Piraccini BM, Sacco C, et al. Taxane-induced nail changes: incidence, clinical presentation and outcome. Ann Oncol. 2003;14(2):333-337.

8. Robert C, Sibaud V, Mateus C, Verschoore M, Charles C, Lanoy E, et al. Nail toxicities induced by systemic anticancer treatments. Lancet Oncol. 2015;16(4):e181-e189. doi: 10.1016/S1470-2045(14)711337

9. Winther D, Saunte DM, Knap M, Haahr V, Jensen AB. Nail changes due to docetaxel-a neglected side effect and nuisance for the patient. Support Care Cancer. 2007;15(10):1191-1197.

10.Ghetti E, Piraccini BM, Tosti A. Onycholysis and subungual haemorrhages secondary to systemic chemotherapy (paclitaxel). J Eur Acad Dermatol Venereol 2003;17(4):459-460. 